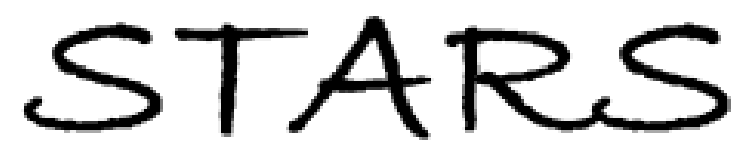

University of Central Florida

STARS

$1-1-2010$

\title{
Design, implementation, and testing of a cryogenic loading capability on an engineering neutron diffractometer
}

\author{
T. R. Woodruff \\ University of Central Florida \\ V. B. Krishnan \\ University of Central Florida \\ B. Clausen \\ T. Sisneros \\ V. Livescu
}

See next page for additional authors

Find similar works at: https://stars.library.ucf.edu/facultybib2010

University of Central Florida Libraries http://library.ucf.edu

This Article is brought to you for free and open access by the Faculty Bibliography at STARS. It has been accepted for inclusion in Faculty Bibliography 2010 s by an authorized administrator of STARS. For more information, please contactSTARS@ucf.edu.

\section{Recommended Citation}

Woodruff, T. R.; Krishnan, V. B.; Clausen, B.; Sisneros, T.; Livescu, V.; Brown, D. W.; Bourke, M. A. M.; and Vaidyanathan, R., "Design, implementation, and testing of a cryogenic loading capability on an engineering neutron diffractometer" (2010). Faculty Bibliography 2010s. 941.

https://stars.library.ucf.edu/facultybib2010/941

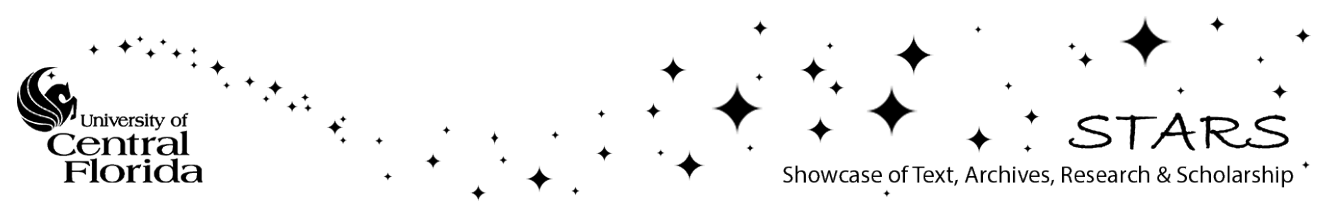




\section{Authors}

T. R. Woodruff, V. B. Krishnan, B. Clausen, T. Sisneros, V. Livescu, D. W. Brown, M. A. M. Bourke, and R. Vaidyanathan 


\section{Design, implementation, and testing of a cryogenic loading capability on an engineering neutron diffractometer}

T. R. Woodruff, V. B. Krishnan, B. Clausen, T. Sisneros, V. Livescu, D. W. Brown, M. A. M. Bourke, and R. Vaidyanathan

Citation: Review of Scientific Instruments 81, 063903 (2010); doi: 10.1063/1.3436637

View online: https://doi.org/10.1063/1.3436637

View Table of Contents: http://aip.scitation.org/toc/rsi/81/6

Published by the American Institute of Physics

\section{Articles you may be interested in}

Sample environment for neutron scattering measurements of internal stresses in engineering materials in the temperature range of $6 \mathrm{~K}$ to $300 \mathrm{~K}$

Review of Scientific Instruments 88, 025103 (2017); 10.1063/1.4974815

Design and implementation of a multiaxial loading capability during heating on an engineering neutron diffractometer

Review of Scientific Instruments 85, 103901 (2014); 10.1063/1.4896042

Development of a cryogenic load frame for the neutron diffractometer at Takumi in Japan Proton Accelerator Research Complex

Review of Scientific Instruments 84, 063106 (2013); 10.1063/1.4810010

A furnace with rotating load frame for in situ high temperature deformation and creep experiments in a neutron diffraction beam line

Review of Scientific Instruments 83, 053901 (2012); 10.1063/1.4708619

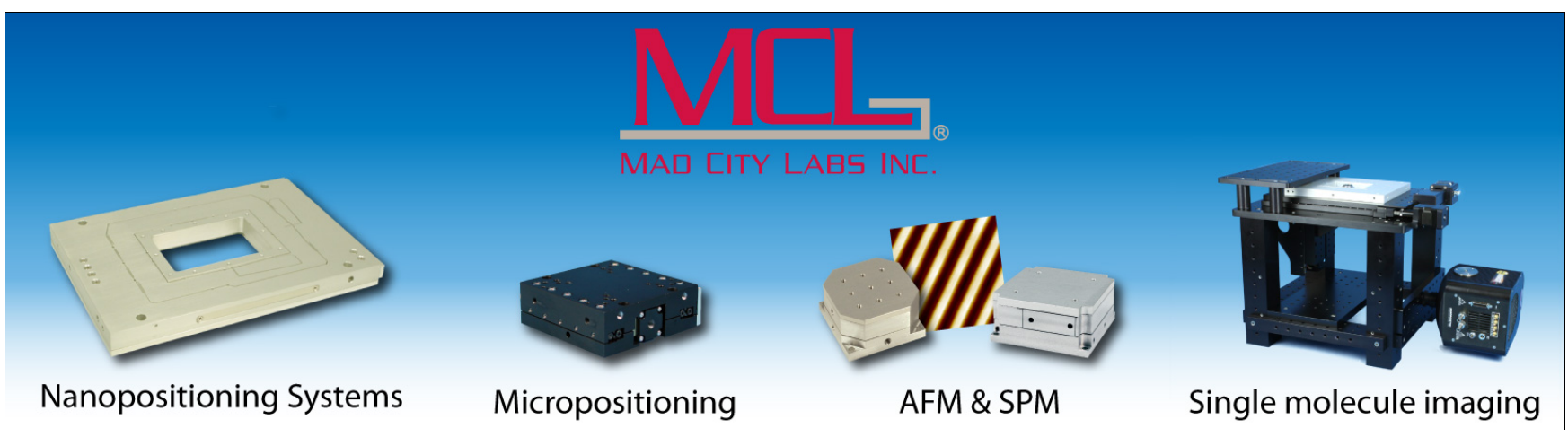




\title{
Design, implementation, and testing of a cryogenic loading capability on an engineering neutron diffractometer
}

\author{
T. R. Woodruff, ${ }^{1}$ V. B. Krishnan, ${ }^{1}$ B. Clausen, ${ }^{2}$ T. Sisneros, ${ }^{2}$ V. Livescu, ${ }^{2}$ D. W. Brown, ${ }^{2}$ \\ M. A. M. Bourke, ${ }^{2}$ and R. Vaidyanathan ${ }^{1, a)}$ \\ ${ }^{1}$ Department of Mechanical, Materials, and Aerospace Engineering, Advanced Materials Processing and \\ Analysis Center (AMPAC), University of Central Florida, Orlando, Florida 32816, USA \\ ${ }^{2}$ Los Alamos National Laboratory, Los Alamos, New Mexico 87545, USA
}

(Received 6 December 2009; accepted 2 May 2010; published online 23 June 2010)

\begin{abstract}
A novel capability was designed, implemented, and tested for in situ neutron diffraction measurements during loading at cryogenic temperatures on the spectrometer for materials research at temperature and stress at Los Alamos National Laboratory. This capability allowed for the application of dynamic compressive forces of up to $250 \mathrm{kN}$ on standard samples controlled at temperatures between 300 and $90 \mathrm{~K}$. The approach comprised of cooling thermally isolated compression platens that in turn conductively cooled the sample in an aluminum vacuum chamber which was nominally transparent to the incident and diffracted neutrons. The cooling/heat rate and final temperature were controlled by regulating the flow of liquid nitrogen in channels inside the platens that were connected through bellows to the mechanical actuator of the load frame and by heaters placed on the platens. Various performance parameters of this system are reported here. The system was used to investigate deformation in Ni-Ti-Fe shape memory alloys at cryogenic temperatures and preliminary results are presented. () 2010 American Institute of Physics.

[doi:10.1063/1.3436637]
\end{abstract}

\section{INTRODUCTION}

Diffraction based techniques for microstructural and micromechanical characterization typically use electrons and $\mathrm{x}$ rays from laboratory sources and are hence limited to probing depths of several microns (at most) below the sample surface. Neutrons, on the other hand, can penetrate several millimeters (and even centimeters in some cases) enabling measurements from polycrystalline samples that are representative of bulk behavior. The neutron diffraction technique can thus be used to obtain information such as crystal structures, residual and internal strains, phase fractions, and texture, among others, from bulk polycrystalline samples. ${ }^{1,2}$ The case of shape memory alloys is one such example wherein the sample surface is not necessarily representative of bulk properties due to surface preparation or stress relaxation effects that can result in a martensitic transformation or localized detwinning. ${ }^{3}$

The neutron diffraction technique is additionally insightful when performed in an in situ mode, i.e., when diffraction measurements are carried out as the sample is stressed, heated, or cooled. While the capability to mechanical load, heat, and diffract has existed at various neutron sources, ${ }^{4}$ there have been limited dedicated capabilities that have facilitated neutron diffraction during cooling and mechanical loading. The objective of this paper is to report on the successful design, implementation, and testing of such a capability on an engineering neutron diffractometer at a spallation neutron source. To the best of the authors' knowledge, this is

\footnotetext{
${ }^{a)}$ Electronic mail: raj@mail.ucf.edu.
}

the first time such a capability has been implemented and reported. The immediate motivation for developing this capability was to initiate two investigations that required use of such a capability. The first was to investigate deformation mechanisms in $\mathrm{Ni}-\mathrm{Ti}-\mathrm{Fe}$ shape memory alloys. There has been renewed interest in the development of low-temperature $\mathrm{Ni}-\mathrm{Ti}-\mathrm{Fe}$ shape memory alloys for various cryogenic actuator applications, such as thermal conduction switches, valves, and seals. ${ }^{5-7}$ These alloys exhibit a fully reversible thermoelastic phase transformation between a hightemperature cubic (B2) so-called austenite phase, an intermediate trigonal so-called $\mathrm{R}$ phase, and a low-temperature monoclinic $\left(B 19^{\prime}\right)$ so-called martensite phase. These phase transformations allow these materials to recover their original shape (shape memory effect) during heating against large stresses up to $500 \mathrm{MPa}$ after being deformed up to $1 \%$ in the $\mathrm{R}$ phase and $8 \%$ in the monoclinic phase). ${ }^{8}$ Neutron diffraction experiments were to be performed on a $\mathrm{Ni}-\mathrm{Ti}-\mathrm{Fe}$ alloy during loading and cooling to $90 \mathrm{~K}$ to follow texture, strain, and phase fraction changes responsible for shape memory behavior. The second reason was to perform residual stress measurements in an INCONEL 718 flow liner used in NASA's space shuttle. Cracks were noted propagating from apertures that were punched in these flow liners that feed liquid hydrogen to the space shuttle engines. ${ }^{9}$ Welding was adopted as a repair route and neutron diffraction needed to be used to study the residual strain fields at cryogenic temperatures in the flow liner and in model coupons prior to and postwelding.

While the aforementioned two studies provided an immediate need for implementing this in situ cryogenic loading 
capability, additional motivation was provided by the desire to make this capability available for future investigations of other engineering materials. These include an understanding of deformation processes in rare earth intermetallics, low symmetry metals, transformation-induced plasticity, and martensitic steels where alternate modes such as strain- and temperature-induced phase transformations and twinning become increasingly dominant at lower temperatures and can hence be conveniently probed in situ. Other candidate systems include composite or multiphase materials where thermal mismatch stresses (arising from the coefficient of thermal expansion mismatch between the constituent phases or anisotropy associated with crystalline symmetry) can increase with decreasing temperatures and make for interesting scientific and engineering studies. ${ }^{10}$

\section{NEUTRON SPECTROMETER FOR MATERIALS RESEARCH AT TEMPERATURE AND STRESS}

Neutron diffraction is commonly used for microstructural and micromechanical characterization of engineering materials and several reviews are available in the literature (e.g., Refs. 1 and 2). Mechanical characterization using neutron diffraction relies on using atomic planes in samples as internal strain gauges. In addition to strain information, the integrated intensity of diffraction peaks in spectra can be used to determine the volume fraction of the scattering phase. Furthermore, the relative intensity of diffraction peaks in a particular phase can be used to determine the amount of texture or preferred orientation in that phase. Such diffraction measurements when performed in situ, i.e., by simultaneously recording diffraction spectra from samples that are subjected to external stresses or temperature changes, or a combination thereof, thus provide quantitative, phase specific information on the evolution of strains, texture, and phase volume fractions.

The neutron spectrometer for materials research at temperature and stress or SMARTS is a third generation neutron diffractometer optimized for the study of engineering materials at Los Alamos National Laboratory. ${ }^{11}$ SMARTS has an extensive array of in situ capabilities for sample environments with measurements on small $\left(1 \mathrm{~mm}^{3}\right)$ or large $\left(1 \mathrm{~m}^{3}\right)$ samples. The high temperature vacuum furnace and load frame allow for research on materials under high loads (250 $\mathrm{kN})$ and high temperatures $(1773 \mathrm{~K})$. This cryogenic loading capability, implemented in this paper, has expanded the capabilities of the SMARTS diffractometer to perform in situ neutron diffraction measurements at temperatures as low as $90 \mathrm{~K}$ during loading. SMARTS has two banks of neutron detectors (each comprising an array of He3 tubes) — bank 1 oriented for measurements from lattice planes whose normals are perpendicular to the loading axis and bank 2 oriented for measurements from lattice planes whose normals are parallel to the loading axis.

\section{DESIGN AND IMPLEMENTATION OF THE CRYOGENIC CAPABILITY}

\section{A. Requirements}

While the technical objective of this work was straightforward, i.e., to develop a capability to facilitate neutron diffraction measurements on polycrystalline samples while simultaneously subjecting them to loads as high as $250 \mathrm{kN}$ (maximum load rating on the frame) and temperatures as low as $90 \mathrm{~K}$ (lowest temperature achievable), there were several implicit accompanying requirements and challenges that needed to be met. These are highlighted in the following.

(i) An unobstructed line of sight for the incident and diffracted neutrons needed to be made available with respect to the mechanically loaded and cooled sample, so that the acquired neutron spectra was not altered in any way (e.g., by changes in peak profiles, peak intensities, background profiles, or introduction of additional peaks, etc.).

(ii) The sample had to be contained in a vacuum in order to prevent freezing and crystallization of moisture around the cold sample that could introduce artifacts in the diffraction spectra.

(iii) The materials used in the load train needed to be resistant to brittle fracture given the high stresses applied at low temperature.

(iv) The design needed to thermally isolate the cooling system from the heat generated by the servohydraulic actuator on the existing SMARTS load frame.

(v) The sample had to be maintained and controlled at the user-specified temperature without any significant temperature fluctuations or spatial temperature gradients.

(vi) The response to a user-specified temperature had to be reasonably rapid in order to optimize the usage of neutron beam time.

\section{B. Design progression and modeling}

The first effort on SMARTS for neutron diffraction at cryogenic temperatures started with the aforementioned project for quantitatively assessing residual strains and stresses associated with the weld repair process used to fix cracks on NASA's space shuttle flow liners. The neutron diffraction measurements were conducted to determine residual strains at selected spatial locations in a welded coupon at 293 and $135 \mathrm{~K}$. The coupons used in this investigation were made of the same INCONEL 718 alloy used for the flow liners and subjected to identical welding and certification procedures that were carried out on the space shuttle. ${ }^{9} \mathrm{~A}$ custom aluminum vacuum chamber (Fig. 1) was fabricated with the objective of conductively cooling the coupons using liquid nitrogen and subsequently integrated on to the SMARTS load frame. The coupon was held in a rectangular frame that was in thermal contact with a copper "cold mass," which served as a heat sink, at the bottom of the vacuum chamber. The temperature of the coupon was controlled by controlling the flow of liquid nitrogen through the copper cold mass. 




FIG. 1. (Color online) Custom aluminum vacuum chamber with a copper cold mass for conductively cooling INCONEL 718 coupons (SMARTS load frame not shown).

The aluminum vacuum chamber for residual strain measurements in the coupons was fabricated with the foresight that it could be used for in situ loading at cryogenic temperatures, performed in conjunction with neutron diffraction. The incident, diffracted, and transmitted beams entered and exited via four rectangular windows, at $90^{\circ}$ to each other and positioned at $45^{\circ}$ with respect to the flanged cylindrical ports. Finite element analysis was used to optimize the thickness of the neutron beam windows (Fig. 2) so as to minimize

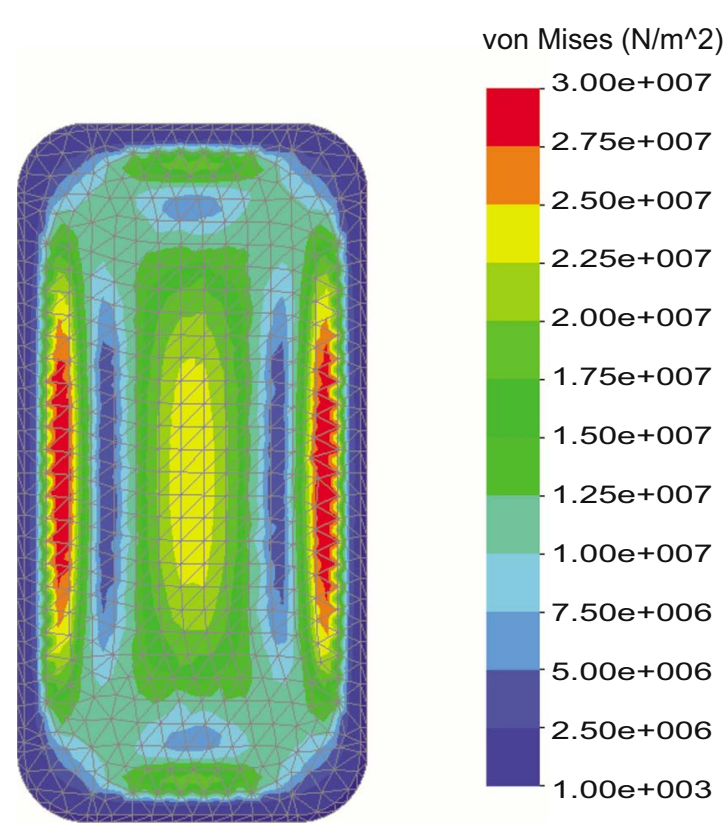

FIG. 2. (Color online) Stress contour plot from finite element model of window in aluminum chamber for passage of the incident, diffracted, and transmitted neutrons while minimizing neutron absorption and diffraction. The plot shows von Mises stresses (in $\mathrm{Pa}$ ) resulting from a differential pressure of $1 \mathrm{~atm}$ due to ideal vacuum in the chamber, computed using solid tetrahedral finite elements with nonlinear in-plane stiffening.
TABLE I. Properties of Vascomax C-350 used in the finite element model.

\begin{tabular}{lc}
\hline \hline Property & Value \\
\hline Young's modulus & $200 \mathrm{GPa}$ \\
Poisson's ratio & 0.33 \\
Shear modulus & $75.2 \mathrm{GPa}$ \\
Mass density & $8080 \mathrm{~kg} / \mathrm{m}^{3}$ \\
Ultimate tensile strength & $2359 \mathrm{MPa}$ \\
Yield strength & $2320 \mathrm{MPa}$ \\
Thermal conductivity & $25.3 \mathrm{~W} / \mathrm{m} \mathrm{K}$ \\
Specific heat capacity & $452.3 \mathrm{~J} / \mathrm{kg} \mathrm{K}$ \\
Coefficient of thermal expansion & $11.3 \times 10^{-6} / \mathrm{K}$ \\
\hline \hline
\end{tabular}

any neutron absorption or diffraction by aluminum while avoiding significant deflections due to the vacuum inside the chamber.

Several design iterations were investigated while developing the cooling system for the cryogenic loading capability on the SMARTS diffractometer. One such design consisted of wrapping the compression platens with thin copper tubing through which liquid nitrogen was circulated. The compression platens subsequently cooled the sample by conduction. The load train of this design with a $24 \mathrm{~mm}$ long, $10 \mathrm{~mm}$ diameter cylindrical NiTi compression sample was modeled using SOLIDWORKS software. Published thermal and mechanical properties for Vascomax C-350 (a cobalt strengthened maraging steel with $18 \% \mathrm{Ni}$ ) were used and the SMARTS compression platens and push rods were modeled using their pre-existing geometry. The properties used are outlined in Table I. The Vascomax C-350 material was chosen due to its high compressive yield strength of $2675 \mathrm{MPa}$ which allows the compression platens to safely apply compressive stresses as high as $2000 \mathrm{MPa}$ without permanently deforming the platens. The model was then simplified using the assumption of axial symmetry and meshed using COSMOSWORKS finite element software. A convective boundary condition was applied to the internal surfaces of the copper tubing using a fluid temperature of $77 \mathrm{~K}$ and a convective coefficient of $500 \mathrm{~W} / \mathrm{m}^{2} \mathrm{~K}$. Similarly, a convective boundary condition was applied to the surfaces of the push rods that lie outside of the vacuum chamber using a fluid temperature of $295 \mathrm{~K}$ and a convective heat transfer coefficient of $3 \mathrm{~W} / \mathrm{m}^{2} \mathrm{~K}$. Inside the vacuum chamber, a convective boundary condition was applied to all external surfaces of the load train using a fluid temperature of $295 \mathrm{~K}$ and a convective heat transfer coefficient of $0.1 \mathrm{~W} / \mathrm{m}^{2} \mathrm{~K}$. Radiation heat leak was modeled inside of the chamber by using an emissivity factor of 0.3 and a radiation shape factor of 0.4 . The end of the push rod that was in contact with the load frame's servohydraulic actuator was given a constant temperature boundary condition of $340 \mathrm{~K}$. Similarly, the end of the push rod that was in contact with the load frame's load cell was given a constant temperature boundary condition of $295 \mathrm{~K}$. The model (Fig. 3) predicted that the system would reach a steady state temperature of $89 \mathrm{~K}$ in approximately $1 \mathrm{~h}$. However, after implementing this design, the actual system took about $2.5 \mathrm{~h}$ to reach a steady state temperature of only 215 $\mathrm{K}$. This disparity was attributed to the contact resistance that existed between the copper coils and the compression plat- 


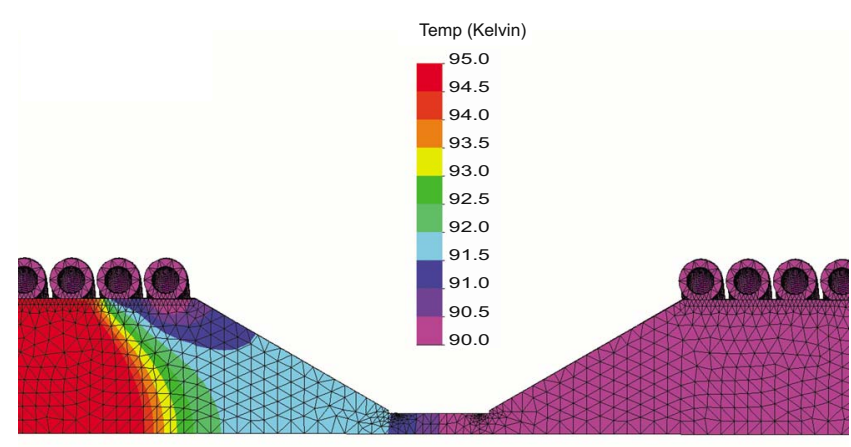

$r^{2}$

FIG. 3. (Color online) Temperature contour plot from finite element heat transfer model of the preliminary cooling system with liquid nitrogen carrying copper tubes wrapped around the platens. The temperatures are in K.

ens. The finite element model assumed perfect contact across the entire length of the copper tubing. However, in reality, the contact was at a few discrete points.

Subsequently, in order to eliminate this contact problem, the cooling system was modified by designing custom compression platens with internal cooling passages through which liquid nitrogen circulated. Additionally, a thermally insulating coupling system was designed to connect the compression platens to the push rods for isolating them from the warm push rods of the SMARTS load frame. As with the previous design concept, the sample was cooled conductively while in contact with the cold compression platens.

\section{Implementation}

The cryogenic capability implemented on SMARTS consists of three main systems: (a) a vacuum system, (b) a cooling system, and (c) a temperature control system. These three systems worked together to maintain the test sample at a specified low temperature between 300 and $90 \mathrm{~K}$. An overall system schematic is shown in Fig. 4. The actual implemen-

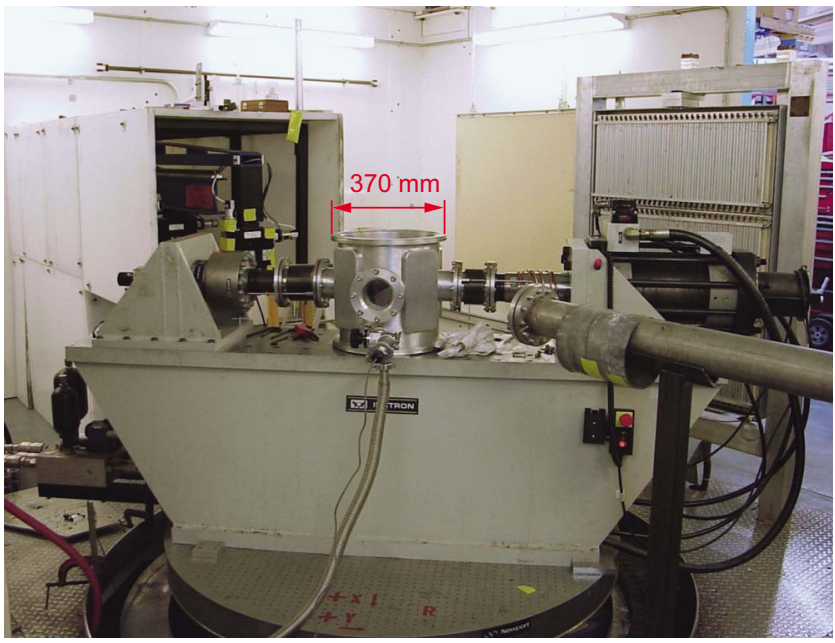

FIG. 5. (Color online) Implementation of the cryogenic loading capability on the SMARTS load frame.

tation on SMARTS is shown in Fig. 5 and discussed in the following.

\section{Vacuum system}

The vacuum system served dual purposes in the cryogenic loading capability. The first purpose was to minimize convective heat losses that would increase the thermal load on the system. The second purpose was to prevent freezing and crystallization of moisture and other gases in the atmosphere at low temperatures. The vacuum system consisted of an aluminum vacuum chamber that enclosed the test sample, two bellows that sealed the load frame's push rods on either side, electrical feedthroughs, liquid nitrogen connections, and a mechanical vacuum pump (Fig. 6). The vacuum chamber was custom made of aluminum, with four rectangular windows oriented at $90^{\circ}$ to each other provided access to the incident, transmitted, and diffracted neutrons. Aluminum was chosen because thin sections of aluminum allow for neutrons

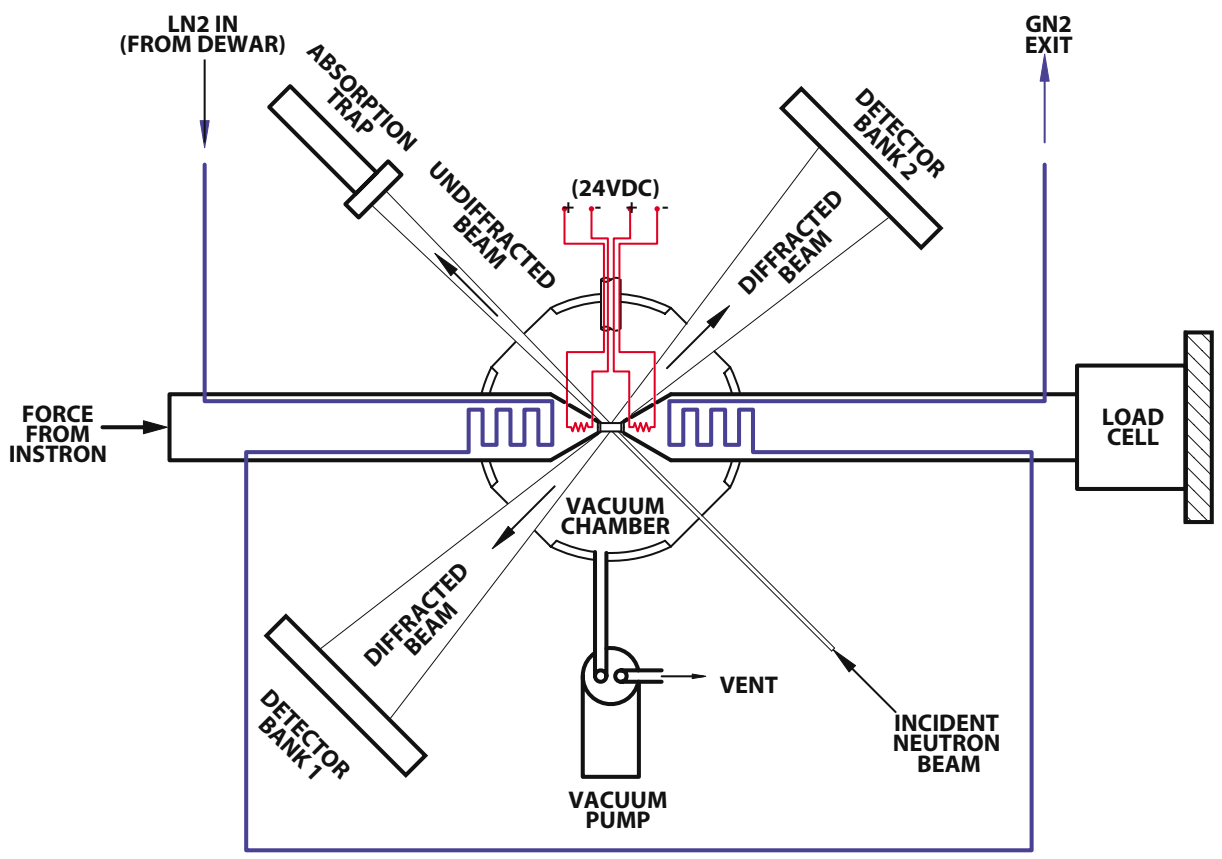

FIG. 4. (Color online) Overall schematic of the cryogenic loading capability implemented. 


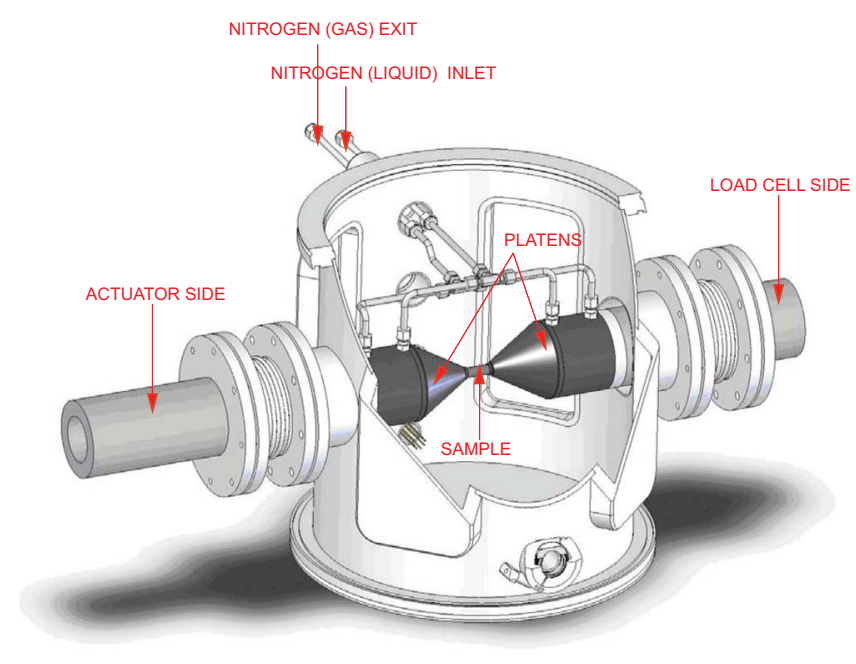

(a)

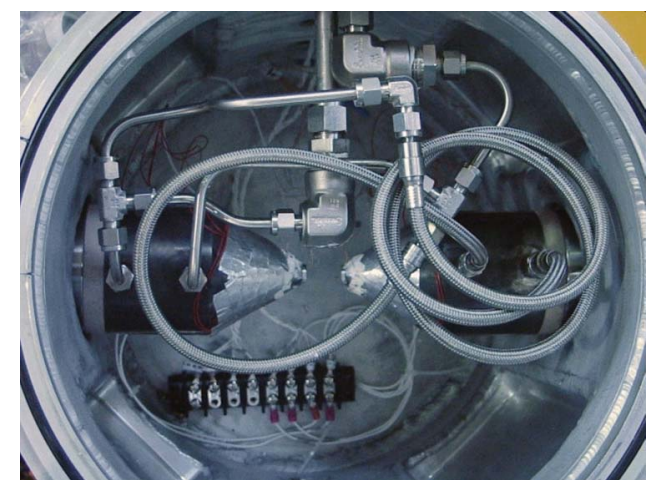

(b)

FIG. 6. (Color online) (a) Design and (b) implementation of the vacuum and cooling system.

to pass through with limited absorption and not introducing additional detectable peaks in the neutron diffraction pattern. Additionally, the choice of aluminum helped reduce the weight of the setup on the $x-y-z-r$ motorized stage. Two identical flanged cylindrical ports that were exactly opposite to each other, positioned at $45^{\circ}$ with respect to the rectangular windows, accommodated the insertion of the two compression platens for loading. The vacuum chamber also incorporated a glass view port that allowed the use of theodolites for aligning the test sample with respect to the incident neutron beam. The two flanged bellows that connected to the two flanged cylindrical ports of the vacuum chamber allowed for smooth movement of the push rods of the SMARTS load frame without introducing frictional forces that compromised the accuracy of the load measurements. These push rods were in turn connected to the platens inside the chamber. The bellows provided an effective seal that isolated the vacuum from the external environment. The vacuum chamber also consisted of vacuum feedthroughs that allowed for the passage of electrical conductors and liquid nitrogen connections through the wall of the vacuum chamber. These feedthroughs were necessary to support the cooling and temperature control systems. A two-stage mechanical pump was used to pull a vacuum in the vacuum chamber of the order of $10^{-4}$ Torr.

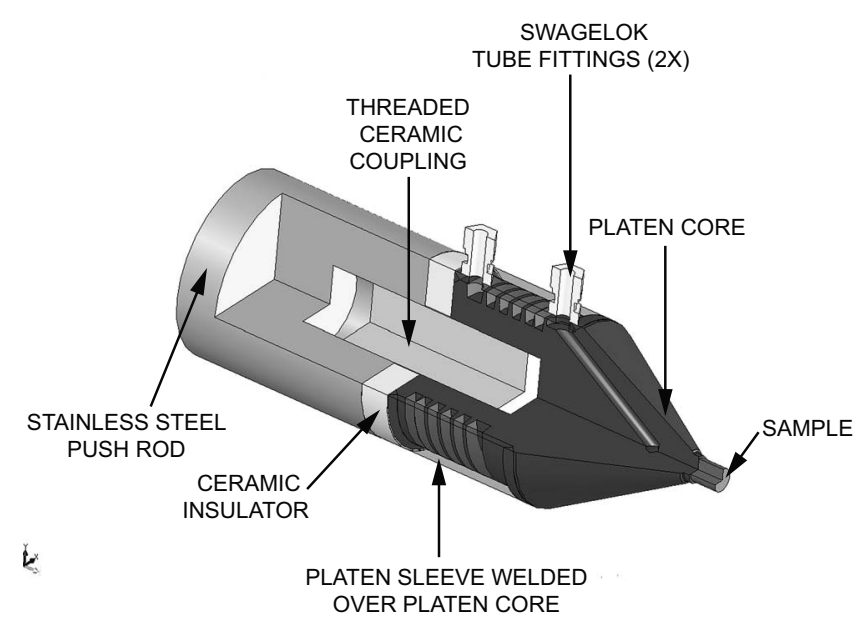

FIG. 7. Model of the compression platens installed on the push rods of the SMARTS load frame.

For safety, a pressure relief valve was included in the vacuum chamber to release any excess pressure, in the event of the circulating liquid nitrogen leaking.

\section{Cooling system}

The cooling system consisted of conductively cooling the test sample via the load applying platens that in turn were cooled by circulating liquid nitrogen through internal cooling passages (Fig. 7). A thermally insulating coupling system isolated the cold custom compression platens from the warm push rods of the SMARTS load frame. The platens were connected to a standard 1601 liquid nitrogen Dewar through fluid transfer lines, flow valves, and vacuum feedthroughs. The cooling passages inside of the custom compression platens first directed the liquid nitrogen into the tip of the compression platen near the test sample. Following flow past the tip of the platen, the liquid nitrogen was returned to the outer periphery of the platen where it flowed through a helical passage that was machined in the compression platen. After flowing through the helical passages in the compression platens, it exited through the exhaust plumbing out of the vacuum chamber. Finite element analysis was used to model the compression platens (Fig. 8) so that the internal cooling passage at the tip of the compression platen could be placed close to the test sample, without causing failure of the cryogenically cooled platen material.

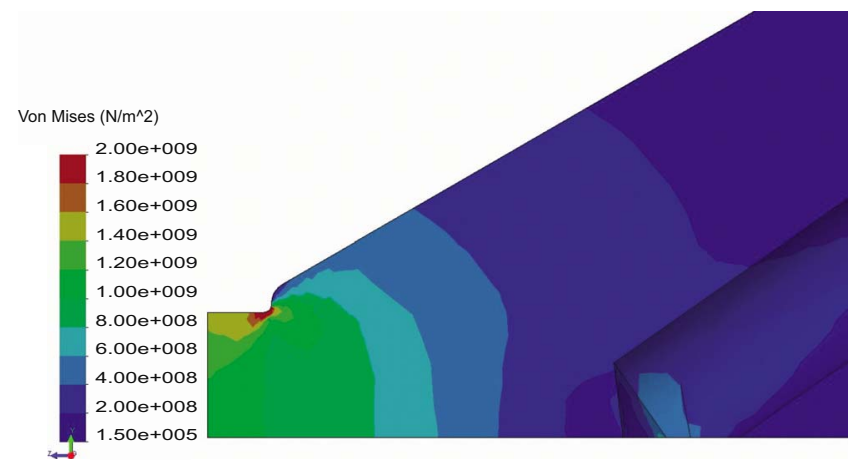

FIG. 8. (Color online) Stress profile at and around the tip of the compression platen from finite element analysis. The stresses are in Pa. 
To increase the efficiency of the cooling system, a coupling system was implemented to thermally isolate the compression platens from the SMARTS load frame. The compression platens were connected onto the push rods using a threaded coupling made of Dupont's Macor ceramic. Furthermore, a ceramic disk separated the abutting faces of the push rods and the compression platens. This formed an insulating layer between the cold compression platens and the warm push rods on the SMARTS load frame. These thermally insulating materials in the vacuum system minimized both conductive and convective heat inputs into the compression platens.

\section{Temperature control system}

The temperature control system helped maintain a constant temperature under dynamic loading and also varied the

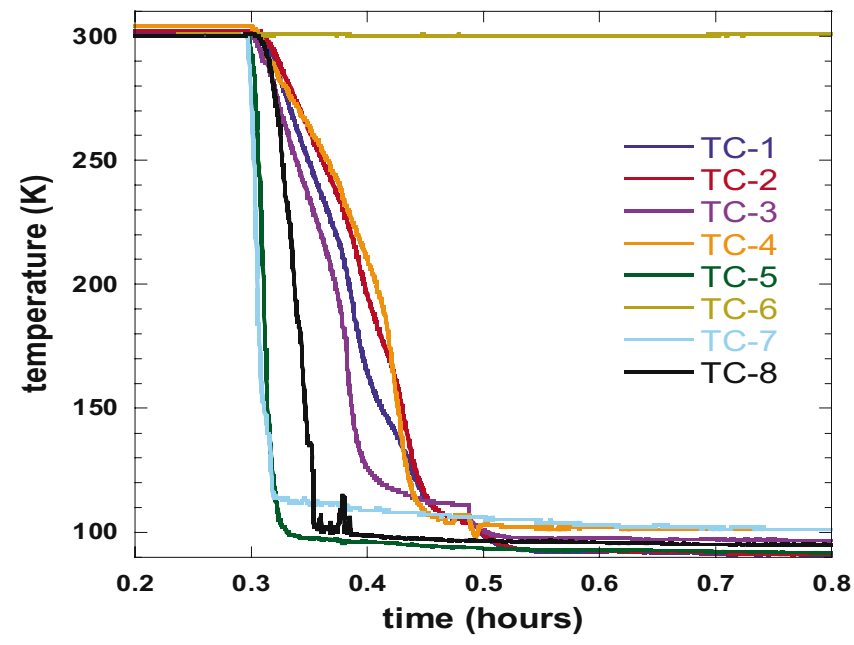

(a)

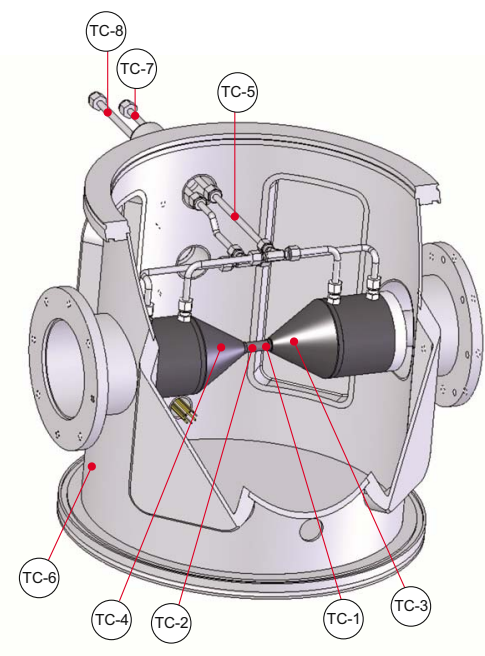

(b)

FIG. 9. (Color online) (a) Cooling performance as observed from thermocouple readings. (b) Schematic showing location of thermocouples. Thermocouples 1-8 represent thermocouples positioned on the load cell side of the sample, actuator side of the sample, platen at the load cell side, platen at the actuator side, inlet of liquid nitrogen connection inside the vacuum chamber, ambient, inlet of liquid nitrogen connection outside the vacuum chamber, and outlet of liquid nitrogen connection outside the vacuum chamber, respectively. temperature at constant load. It consisted of a custom proportional-integral-derivative control program developed using LABVIEW software, a set of surface heaters mounted on the conical surface of each platen and thermocouples mounted at various locations on the test sample, platens, inlet, and vent locations of the liquid nitrogen lines. The controller collected temperature data from the thermocouples and maintained the temperature though surface heaters. The temperature control system allowed the temperature of the test sample to stabilize at any temperature specified by the operator, above or equal to $90 \mathrm{~K}$. This further required manual regulating of liquid flow valves, in certain cases. The surface heaters had two purposes: one, of eliminating the temperature gradient between the platens due to the thermal load mismatch, and two, of warming up the test sample relatively quickly if necessary. The thermal control system had independent power and feedback channels for each of the compression platens, which allowed the operator to set the temperature at each end of the test sample independently.

\section{SYSTEM PERFORMANCE}

The performance of the cryogenic capability was evaluated by using a $\mathrm{Ni}_{46.8} \mathrm{Ti}_{50} \mathrm{Fe}_{3.2}$ shape memory alloy test sample. After circulating liquid nitrogen through the platens, a steady state of $90 \mathrm{~K}$ (sample temperature) was reached in 18 min. Furthermore, the consumption of liquid nitrogen was reduced from $27 \mathrm{l} / \mathrm{h}$ (when circulated with copper tubes around the platens as described previously) to $13.3 \mathrm{l} / \mathrm{h}$ with the current setup. Figure 9(a) shows the cooling performance of the system which shows uniform temperatures in the sample for the duration of a typical neutron experiment. Thermocouples 1-8 represent thermocouples positioned on the load cell side of the sample, actuator side of the sample, platen at the load cell side, platen at the actuator side, inlet of liquid nitrogen connection inside the vacuum chamber, ambient, inlet of liquid nitrogen connection outside the vacuum chamber, and outlet of liquid nitrogen connection outside the vacuum chamber, respectively [as shown in Fig. 9(b)]. It is expected that the temperature across the sample be monitored in a similar manner to ensure the absence of substantial gradients in the sample. This is particularly necessary in the case of samples with low thermal conductivity and the finite element model previously presented (Fig. 3) can assist in evaluating the need for additional cooling. A representative spectrum obtained at $92 \mathrm{~K}$ and under $425 \mathrm{MPa}$ is shown in Fig. 10. There was no detectable evidence of heating during mechanical loading. As described previously, the alloy undergoes a stress-induced $\mathrm{R}$ phase to martensite phase transformation. This martensite phase was captured by recourse to Rietveld refinement ${ }^{8}$ and the absence of any detectable peaks from the aluminum chamber was confirmed. For direct comparison, a spectrum is also shown from the same sample in the austenite or $B 2$ phase at room temperature in the unloaded condition.

\section{CONCLUDING REMARKS}

A cryogenic loading capability wherein the temperature was varied between 300 and $90 \mathrm{~K}$ while collecting neutron 


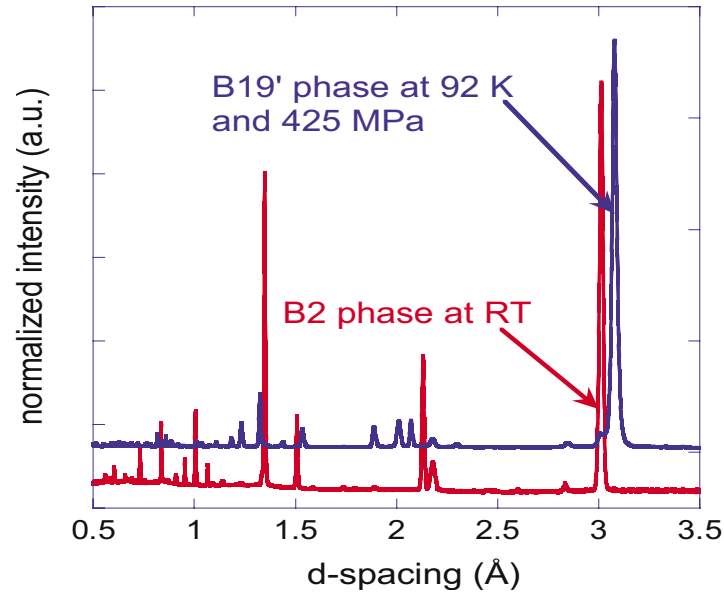

FIG. 10. (Color online) Neutron spectra obtained from a $\mathrm{Ni}_{46.8} \mathrm{Ti}_{50} \mathrm{Fe}_{3.2}$ sample maintained at $92 \mathrm{~K}$ and under an applied compressive stress of 425 $\mathrm{MPa}$. At this temperature and stress, the alloy exhibits a stress-induced phase transformation to the martensite or $B 19^{\prime}$ phase. For direct comparison, a spectrum is also shown from the sample in the austenite or $B 2$ phase at room temperature in the unloaded condition. The spectra shown here are from diffracting planes whose normals are parallel to the loading direction.

diffraction spectra in situ during loading standard samples to $250 \mathrm{kN}$ was successfully installed and tested on the SMARTS diffractometer at Los Alamos National Laboratory. The experimental setup thus expanded the capabilities of the SMARTS diffractometer to include performing in situ neutron diffraction measurements during loading at cryogenic temperatures. Such a capability opens doors for a range of investigations on engineering materials. The capability met implicit requirements of the in situ neutron diffraction technique-by not hindering the path for incident and diffracted neutrons, not causing moisture or atmospheric gases to freeze and crystallize on the test sample, uniformly cooling down the test sample quickly so as to optimize use of neutron beam time, among others. The capability for the most part employed automated control with some manual input through the control of primary flow valves. Future improvements include using pneumatically controlled valves with optimal control parameters so as to lower heating and cooling times and more stable temperature control.

\section{ACKNOWLEDGMENTS}

This work has benefited from the use of the Lujan Neutron Scattering Center at LANSCE, which is funded by the Department of Energy's Office of Basic Energy Sciences. Los Alamos National Laboratory is operated by Los Alamos National Security LLC under DOE Contract No. DE-AC5206NA25396. Financial support from SRI, NASA (Grant No. NAG3-2751), and NSF (Grant No. CAREER DMR0239512) is gratefully acknowledged. The authors are grateful to C. R. Rathod and S. B. Shmalo (University of Central Florida) for valuable experimental assistance.

${ }^{1}$ Measurement of Residual and Applied Stress Using Neutron Diffraction, edited by M. T. Hutchings and A. D. Krawitz (Kluwer Academic, Netherlands, 1992)

${ }^{2}$ M. T. Hutchings, P. J. Withers, T. M. Holden, and T. Orenzen, Introduction to the Characterization of Residual Stress by Neutron Diffraction (CRC, Boca Raton, FL, 2005).

${ }^{3}$ S. Rajagopalan, A. L. Little, M. A. M. Bourke, and R. Vaidyanathan, Appl. Phys. Lett. 86, 081901 (2005).

${ }^{4}$ See: http://www.ncnr.nist.gov/nsources.html for a listing of neutron sources worldwide.

${ }^{5}$ V. B. Krishnan, J. D. Singh, T. R. Woodruff, W. U. Notardonato, and R. Vaidyanathan, Adv. Cryog. Eng. 50A, 26 (2004).

${ }^{6}$ J. L. Lemanski, V. B. Krishnan, R. Mahadevan Manjeri, W. U. Notardonato, and R. Vaidyanathan, Adv. Cryog. Eng. 52A, 3 (2006).

${ }^{7}$ V. B. Krishnan, C. Bewerse, W. U. Notardonato, and R. Vaidyanathan, Adv. Cryog. Eng. 54, 3 (2008).

${ }^{8}$ V. B. Krishnan, R. Mahadevan Manjeri, B. Clausen, D. W. Brown, and R. Vaidyanathan, Mater. Sci. Eng., A 481-482, 3 (2008).

${ }^{9}$ C. R. Rathod, V. Livescu, B. Clausen, M. A. M. Bourke, W. U. Notardonato, M. Femminineo, and R. Vaidyanathan, Adv. Cryog. Eng. 50A, 167 (2004).

${ }^{10}$ V. Livescu, T. R. Woodruff, B. Clausen, T. Sisneros, M. A. M. Bourke, W. U. Notardonato, and R. Vaidyanathan, Adv. Cryog. Eng. 50A, 83 (2004).

${ }^{11}$ M. A. M. Bourke, D. C. Dunand, and E. Ustundag, Appl. Phys. A: Mater. Sci. Process. 74, s1707 (2002). 